\title{
Design tactics in curriculum development: Examples from the Paradigms in Physics ring cycle
}

\author{
Mary Bridget Kustusch, ${ }^{1,{ }^{*}}$ Corinne Manogue, ${ }^{2}$ and Edward Price $\odot^{3}$ \\ ${ }^{1}$ Department of Physics and Astrophysics, DePaul University, \\ 2219 N. Kenmore Avenue Suite 211, Chicago, Illinois 60614, USA \\ ${ }^{2}$ Department of Physics, Oregon State University, 301 Weniger Hall, Corvallis, Oregon 97331, USA \\ ${ }^{3}$ Department of Physics, California State University San Marcos, San Marcos, California 92096, USA
}

(Received 9 July 2019; accepted 7 February 2020; published 4 December 2020)

\begin{abstract}
[This paper is part of the Focused Collection on Curriculum Development: Theory into Design.] A level of curricular design, called design tactics, is identified to fill a gap in the research literature between the broad principles that guide curriculum development and the detailed writing of specific activities and lessons. The use of design tactics is illustrated with a case study using a sequence from middle-division undergraduate electromagnetism, the ring cycle, developed as part of the Paradigms in Physics project at Oregon State University. A retrospective analysis of the curriculum development process identified ten design tactics, related to content, sensemaking, and classroom practice. Further analysis of secondary implementations at DePaul University and California State University San Marcos illustrates how design tactics can also be used to make adaptations to local settings and contexts. Design tactics can serve as a productive bridge between higher-level design principles and activity development.
\end{abstract}

DOI: 10.1103/PhysRevPhysEducRes.16.020145

\section{INTRODUCTION}

Physics educators and physics education researchers have developed and popularized many curricula and instructional techniques (see PhysPort [1] for many examples). Creating curriculum or classroom activities requires decisions about goals, topics, modalities of instruction, and many other factors. In making these decisions, developers' may draw on and respond to learning goals, institutional context, and research on student learning about specific topics. They may also use design principles and models for curriculum development that are based in research on student learning of science and instruction design.

The intent of this paper is to begin to fill a hole in the existing literature-the space between broad design principles that guide the development, refinement, and adaptation of curricular materials. We refer to this missing level as design tactics, which we define as practical instructions that guide developers in designing (and refining) curricular materials in order to meet their specific learning goals. While design tactics do not provide a formula or template for creating activities, they are more specific than design principles. In using the term design tactics, we are drawing

*mkustus1@depaul.edu

Published by the American Physical Society under the terms of the Creative Commons Attribution 4.0 International license. Further distribution of this work must maintain attribution to the author(s) and the published article's title, journal citation, and DOI. from Beach's use of tactics within image theory as "specific, palpable actions that are intended to facilitate implementation of an abstract plan to further progress toward a goal.'[2]

We place design tactics near one end of a spectrum of increasingly practical and concrete ideas from which developers draw in creating curriculum. These ideas range from the research literature (including learning theory), to research-based design principles, to design tactics. For example, curricular materials developed by the physics education research (PER) community have been accompanied by publications describing the research basis for the development of the innovation [e.g., 3,4]. Less frequently, physics curriculum developers have described researchbased principles that guided their curricular design process [e.g., 5,6]. Others within the PER community have described higher-level, more general processes intended to guide faculty in transforming science, technology, engineering, and mathematics (STEM) courses to be more interactive and effective [7].

Figure 1 highlights where we see design tactics fitting with other aspects of curriculum development. The figure identifies a number of contributions to the development, refinement, and adaptation of curricular materials. While many of these elements interact and inform each other, the focus of Fig. 1 is on how they impact the curricular materials. Research on student learning of science and instructional design informs curriculum development models, which describe a general process for development (more on this in Sec. II A). Also informed by broader 


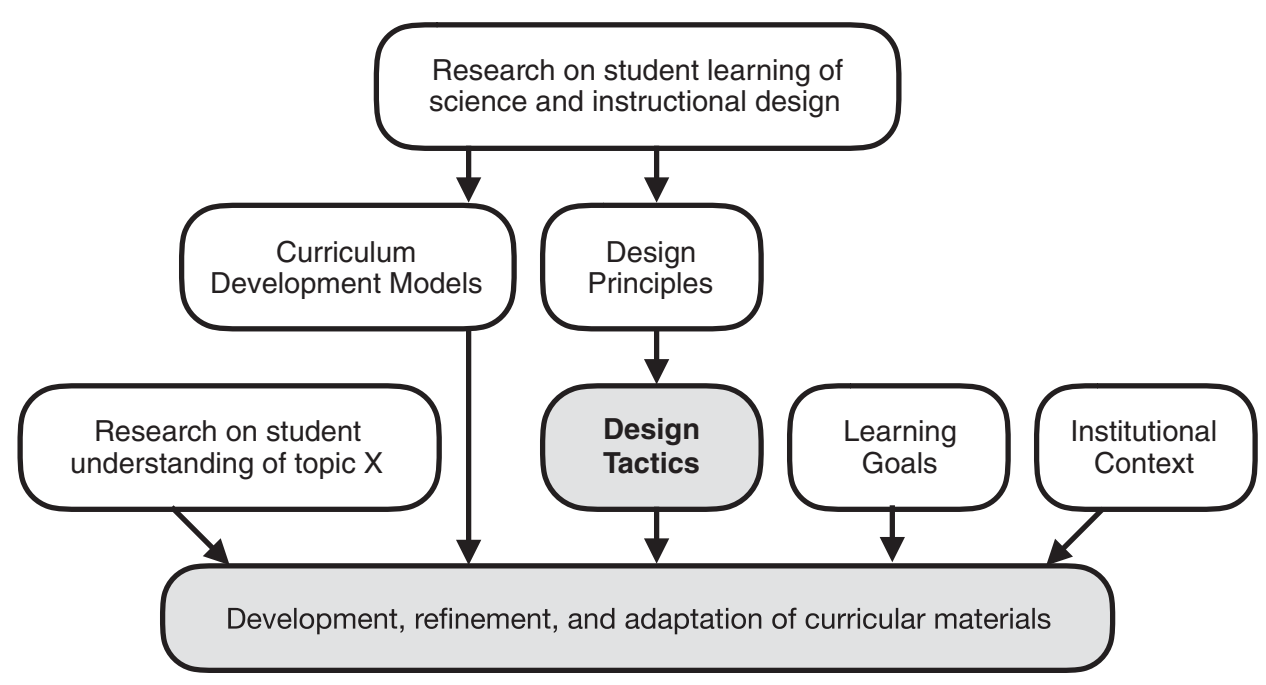

FIG. 1. A model for how design tactics fit within curriculum development. Vertical placement roughly corresponds to the level of abstraction (e.g., curriculum development models are more abstract than learning goals) and arrows represent how the elements impact the curricular materials (e.g., research on learning of topic $X$ can impact development more directly than broader research which is typically mediated through curriculum development models and design principles.

research, design principles and design tactics provide guidelines that are used in parallel with the processes of curriculum development models. Design principles are more general than design tactics, which are more practical and more closely guide specific development processes. However, design tactics must be consistent with the principles. Learning goals and institutional context (including such factors as student population, program structure, course format, etc.) also more directly inform development and adaptation. Finally, developers may directly draw on relevant specific research, for instance, about student understanding of a particular topic. As discussed in Sec. VD, design tactics, learning goals, and institutional context are most directly relevant for secondary implementations.

We developed the concept of design tactics through a retrospective analysis of a curriculum development process. During the course of the analysis, it became clear that the developers had used a number of "guidelines" that were not as high level as design principles. These are what we came to identify as design tactics. While we suspect that many curriculum developers use something similar to design tactics-some systematic processes for translating principles to design - we have not seen examples of these being made explicit and published. In proposing the idea of design tactics, and providing examples from our own curriculum development work, we hope to introduce and formalize this concept and encourage others to be more explicit about their curriculum development process.

Within the context of a specific curricular sequence, this paper addresses the following questions: (i) What design tactics are used to translate general design principles and theories of learning into the design of specific activities and sequences of activities and (ii) what design tactics are used to adapt existing curriculum to new settings, student populations, or course formats?

Our context for addressing these questions is a sequence in upper-division electromagnetic theory called the ring cycle. Author C. A. M. was the lead developer of the original sequence and has taught it multiple times; authors M. B.K. and E. P. have adapted the sequence to their institutions and have also taught it multiple times. Both M. B. K. and E.P. have worked closely with C. A. M. previously during the refinement stages of the development of the ring cycle. In our roles as instructors, we consider this sequence to be particularly successful at meeting our goals for middle-division students.

In this paper, we analyze the original development process and resulting activities for the ring cycle, as well as two secondary implementations. Section II provides background and describes the theoretical perspectives and research base which guided development of the ring cycle. Section III presents the learning goals and activity descriptions for the ring cycle and Sec. IVA provides a brief description of our analysis methods and institutional settings. Section V is the main analysis section of the paper. Here we identify the design tactics and describe how they were employed in developing the activities to accomplish our learning goals. Further, for the secondary implementations, we identify how the design tactics were used to adapt the activities to different settings. In Sec. VI, we discuss how these design tactics interact with each other and with ideas in the broader PER community. Finally, we provide some conclusions about the possible role of these design tactics in Sec. VII.

We hope that the concept of design tactics as a bridge between research, higher-level design principles, and actual activity development will be useful to other curriculum 
developers. Further, we expect that the specific design tactics described here may be useful to those creating activities for similar contexts or adapting materials for secondary implementations as well as serve as an example and starting point for other curriculum developers to articulate and share their own design tactics.

\section{BACKGROUND}

\section{A. Research on curriculum development}

As described above, curriculum development is informed by a range of ideas and the question of what to include in curriculum and how to develop it has been addressed in various degrees of specificity by different research traditions and researchers.

The field of instructional design presents several models that are specifically intended to guide the process of instructional design. However, these are generally very high-level models. ADDIE is a well-known example [8] consisting of sequential, but iterative stages of analysis, design, development, implementation, and evaluation. The PER literature also includes examples of high-level processes for curriculum design. The science education initiative (SEI) model for research-based course transformation is one example, and is based on a process of identifying outcomes or goals, assessment, and curricular design [7]. In characterizing ADDIE and the SEI approach as high-level models, we mean that while they prescribe an overall process, they do not guide the development of specific classroom activities. ADDIE in particular does not include choices about instructional format or pedagogy. The SEI model advocates for research-based instructional practices and interactive techniques, but is also a fairly general model.

Design principles, on the other hand, represent a particular stance or set of commitments about student learning, teaching, and the organization of instructional settings that provide high-level guidance for decision making. Design principles are distillations of research on students' learning of science that are formulated in a way to guide the development of curriculum. Some developers of curricula for university-level physics courses have described the principles that guided their design process $[5,6]$. For example, one of Goldberg et al.'s design principles is "learning builds on prior knowledge" [5]. The activities in their curriculum have a standard structure, and one section, called initial ideas, "is designed to elicit students' prior knowledge about the central issue of the activity." [5] The individual initial ideas sections in each specific activity take into account the principle that learning builds upon prior knowledge; however, they may include different class activities (small group discussions, whole class discussions, concept questions, etc.) and with different content topics. Other design principles recognize the role of interactions with peers and tools, but the general design principles do not provide guidance for specific choices at the level of activity development.

\section{B. Research contributing to the design of the Paradigms}

The Paradigms in Physics project [9-11] at Oregon State University (OSU) is the broad context for the work presented in this paper. The Paradigms are an ongoing $20+$ year effort to radically revise the middle- and upperdivision physics courses for physics majors and their development was informed by a range of theoretical perspectives and research findings. The Paradigms include revised content at both the program and course levels. At the program level, topics are rearranged to better reflect the way professional physicists think about the field. At the course level, topics are resequenced to better support student learning. The Paradigms incorporate a variety of pedagogical approaches featuring small group work where students actively engage with physics content.

An early articulation of the Paradigms approach, which is exemplified in the ring cycle, stated

In brief, PER has taught us that a number of factors will improve student learning: active participation (as opposed to passive note-taking) in exploring the material; a spiral approach that returns to common themes with an increasing level of sophistication or complexity; exploring examples before discussing the general theory; group activities and peer instruction, in which students work with one another during class time in response to problems or exercises posed by the instructor; and a clear focus on content objectives, which forces instructors to trim or repackage bloated courses to accommodate what they reasonably expect students to master in the allotted time-sometimes called the "less is more" approach [11].

Further articulation of this approach has developed concurrently with the curriculum in response to the developers' experience teaching students in the middle division; to research on the Paradigms curriculum [12-26]; and in response to conversations with and the research of the broader PER community [27,28]. For more information, a formal study [29] details the history of the Paradigms including the development of principles and goals.

Development of the Paradigms was guided by findings from research on students' learning of science, such as that students need to be actively engaged in building their understandings [30]. The on-going research on student learning conducted within the Paradigms in Physics program has contributed to the studies in upper-division contexts called for in reviews of discipline-based education research [31] and focused collections [32]. Our emphasis on group activities and peer instruction is consistent with social-constructivist theories of learning [33] and, in 
particular, with attention to how students' understandings evolve as they participate in small and large group activities and discussions [34]. In sociocultural theories, learning occurs through interactions with others and with our environment through tools [e.g., 35,36]. This is consistent with one of the most robust findings in physics education research: the importance of active engagement for learning $[37,38]$. The value of actively engaging students is central in our approach to designing learning environments and activities. In addition to engaging the mind, it is also important to engage the body through kinesthetic or embodied learning activities [39].

Development of the Paradigms was also informed by perspectives from cognitive science [40] and distributed cognition [41]. Cognitive structures such as mental schema (discussed in the context of physics education by Redish [40]) and concept images [42] suggest that "in-the-brain" experiences build both new nodes in the concept image and new connections that increase the chance that nodes will fire together. The ideas of short-term or working memory and "chunking" [43] call attention to the challenges learners face when encountering a symbolically rich, complex topic such as electromagnetism. Distributed cognition calls our attention to the way thinking can be embedded in and mediated by tools such as representational forms, whiteboards, and other manipulables. In addition, the Paradigms have also drawn on the use of cognitive task analysis [44] to explore how experts approach the tasks that we ask of students in order to better connect what is done in the classroom to the goal of students becoming physicists [45].

The Paradigms development team recognizes that the emphasis on social interactions in a social-constructivist theory of learning requires that attention be paid to the establishment of norms around what types of interactions are to be expected and how those interactions will be facilitated [46]. Vygotsky's zone of proximal development (ZPD) [33], which is often described as the region between what learners can do with and without help, suggests the need to be aware of learners' current level of understanding (which varies by student and over time) and match the difficulty of tasks and feedback accordingly, often through scaffolding $[47,48]$. Keeping course activities within the ZPD of as many students as possible is an important commitment among the Paradigms development team. We also believe that it is the moral responsibility of teaching teams to work toward an equitable learning environment, where we use Esmonde's definition of equity as "a fair distribution of opportunities to learn or opportunities to participate" [49].

\section{Research on secondary implementations: Adoption and adaptation}

Curriculum development often takes place at a single or small group of institutions. While there are many similarities among U.S. university physics courses and programs, differences in course format, instructor preferences, student background, and other characteristics may limit the relevance of curricula developed in other settings, or require adaptations to make them effective. One focus of this paper is to consider how design tactics may be useful in making these types of changes. Previous work in PER has studied how and why faculty do or do not take up existing researchbased instructional practices [50-55]. Henderson and Dancy [56] introduced an adoption-adaptation-reinvention continuum to describe the varying degrees of change faculty make in educational innovations they decide to implement. Many faculty modify existing innovations in ways large (reinvention) and small (adaptation) [53]. Often, changes result in practices that lack key elements of the original innovation, and may therefore not have outcomes comparable to the original $[50,52,57]$. However, Henderson and Dancy also found that some faculty make changes in order to accommodate local circumstances or instructor preferences, but are consistent with the original core pedagogical principles [50]. In this paper, we are interested in whether and how design tactics are used to adapt innovations, especially when they are used at different types of institutions, different course formats, or with different student populations than in the original development.

\section{THE RING CYCLE}

The ring cycle [58] is a sequence of activities designed for an upper-division course in electromagnetism covering at least electro- and magnetostatics (Chaps. 1, 2, and 5 of Griffiths's electromagnetism textbook [59]). The ring cycle was designed with the assumption that students may have previously had varied and inconsistent experience with vector calculus in mathematics courses and a fairly typical treatment of electromagnetism, using only trivial 1D integration, in a lower-division calculus-based physics course.

\section{A. Learning goals}

The primary learning goal for the original development of the Paradigms, as articulated in the original grant proposal, was for students to "learn to think like a physicist" $[18,19,23]$. Although this goal is recognizable and often articulated by traditional physicists, it is typically unclear what exactly is meant by it, or how to achieve it.

In Table I, we identify the learning goals for the ring cycle that represent one way to refine and unpack what we mean by "thinking like a physicist," at least for this slice of curriculum. These goals are grouped into two broad categories: content goals and sensemaking goals. We use sensemaking as "seeking coherence between different representations of physics knowledge" [60].

While all of the learning goals listed in Table I are learning goals of the ring cycle as discussed in this paper, some of these goals (S7, C6) are independent and could be omitted in a different curricular structure. 
TABLE I. The learning goals associated with the ring cycle.

Content goals: Students should be able to

$\mathrm{C} 1$ - write vectors in different coordinate systems,

C2-use curvilinear coordinates to calculate electrostatic and magnetostatic fields from source distributions with high symmetry,

C3 - identify and appropriately treat line, surface, and volume sources,

C4 - calculate fields at ANY point in space, not just at points with high symmetry,

C5 - calculate fields at points with high symmetry as a limiting case,

C6 - calculate fields by building other sources out of pieces you have (i.e., cylinder out of ring).

Sensemaking goals: Students should be able to

$\mathrm{S} 1$ - make professional choices such as such as deciding which representation(s) to use or what approach to take to a problem,

S2 - utilize and coordinate between multiple representations,

S3 - distinguish between position vectors for the source and field points in the distance formula,

S4 - explore the relationship between discrete and continuous source distributions,

S5 - relate given information to the variables in a formula, also described as employing the "use what you know" strategy. The strategy is particularly apt for evaluating integrals where physicists, including students, must use information about $d \vec{r}$ in curvilinear coordinates to evaluate the integration measure,

S6 - understand integration as using the superposition principle by chopping up the source and adding up the various contributions to the field,

S7 - recognize that not all integrals can be done in closed form,

S8 - recognize that integrating a vector field expressed in rectangular basis vectors (only) is simply the sum of three integrals.

\section{B. Activities}

There are ten discrete, coherent, and well-structured activities in the ring cycle, which play out over several weeks of instruction. They are connected and supported, as necessary, with mini lectures, whole class discussions, and questions that students answer on individual small whiteboards. See Table II for a short description of each activity, the learning goals that are addressed by the activity, the new ideas that are introduced in the activity, and, if relevant, the iconic equation used in the activity.

It is important to note that this sequence does not sit in isolation. There are a number of other activities and

TABLE II. The activities of the ring cycle including: name, type of activity, learning goals addressed, key new ideas introduced in the activity, a short description of the activity and iconic equation, if applicable.
A1: Star Trek
Type:
Kinesthetic
Goals:
S1, S2, S3
Key new ideas:
Description:
Distance formula, origin
A short improvisational role-playing skit based on the Star Trek series in which students explore the definition and notation for position vectors and the geometric nature of the distance formula.

$$
\left|\vec{r}-\vec{r}^{\prime}\right|=\sqrt{\left(x-x^{\prime}\right)^{2}+\left(y-y^{\prime}\right)^{2}-\left(z-z^{\prime}\right)^{2}}
$$

\section{A2: Electrostatic potential due to two point charges}

Type: $\quad$ Small group

Goals: $\quad$ S3, C4

Key new ideas: $\quad$ Superposition, symmetry

Description: $\quad$ A small group activity in which students use the superposition principle to find the electrostatic potential $V$ everywhere in space due to a pair of charges (either identical charges or a dipole). The whole class wrap-up discussion then compares and contrasts the results and discuss the symmetries of the two cases.

$$
V(\vec{r})=\frac{1}{4 \pi \epsilon_{0}} \sum_{i} \frac{q_{i}}{\left|\vec{r}-\vec{r}_{i}\right|}
$$


TABLE II. (Continued)

\section{A3: Distance formula in curvilinear coordinates}

Type: Homework

Goals: $\quad$ S2, S3, C1

Key new ideas: $\quad$ Curvilinear coordinates

Description: $\quad$ A homework problem in which students find the distance between two points $\left|\vec{r}-\vec{r}^{\prime}\right|$ in rectangular, cylindrical, and spherical coordinates.

$$
\begin{aligned}
\left|\vec{r}-\vec{r}^{\prime}\right| & =\sqrt{s^{2}+s^{\prime 2}-2 s s^{\prime} \cos \left(\phi-\phi^{\prime}\right)+\left(z-z^{\prime}\right)^{2}} \\
& =\sqrt{r^{2}+r^{\prime 2}-2 r r^{\prime}\left[\sin \theta \sin \theta^{\prime} \cos \left(\phi-\phi^{\prime}\right)+\cos \theta \cos \theta^{\prime}\right]}
\end{aligned}
$$

A4: Charge density

Type:

Goals:

Kinesthetic

S1, S2, S4, S5, S6

Key new ideas:

Description:

Charge density (linear $\lambda$, surface $\sigma$, volume $\rho$ )

A kinesthetic activity in which students, representing point charges, are asked to act out linear $\lambda$, surface $\sigma$, and volume $\rho$ charge densities and discuss what it means to measure these quantities.

A5: Electrostatic potential due to a ring of charge

Type: $\quad$ Small group

Goals: $\quad$ S2, S4, S5, S6, S7, C2, C3, C4, C5

Key new ideas: Integration, continuous vs. discrete distribution, linear vs. volume elements

Description: $\quad$ A small group activity in which students find the electrostatic potential, $V$, everywhere in space, due to a ring of charge with radius $R$ and total charge $Q$.

$$
V(\vec{r})=\frac{1}{4 \pi \epsilon_{0}} \int \frac{\rho\left(\vec{r}^{\prime}\right)}{\left|\vec{r}-\vec{r}^{\prime}\right|} d \tau^{\prime}
$$

A6: Electric field due to a ring of charge

Type: $\quad$ Small group

Goals: $\quad$ S2, S4, S5, S6, S7, S8, C2, C3, C4, C5

Key new ideas: $\quad$ Vector in numerator, curvilinear basis vectors

Description: $\quad$ A small group activity in which students find the electric field, $\vec{E}$, everywhere in space, due to a ring of charge with radius $R$ and total charge $Q$.

$$
\vec{E}(\vec{r})=\frac{1}{4 \pi \epsilon_{0}} \int \frac{\rho\left(\vec{r}^{\prime}\right)\left(\vec{r}-\vec{r}^{\prime}\right)}{\left|\vec{r}-\vec{r}^{\prime}\right|^{3}} d \tau^{\prime}
$$

\section{A7: Steady current/current density}

Type: Kinesthetic

Goals: $\quad$ S1, S2, S4, S6, C3

Key new ideas: $\quad$ Steady current, current density (linear $\vec{I}$, surface $\vec{K}$, volume $\vec{J}$ )

Description: $\quad$ A kinesthetic activity in which students, representing point charges, are asked to move so as to make an imaginary magnetic field meter register a constant magnetic field, introducing "steady current" as a concept. Students act out linear $\vec{I}$, surface $\vec{K}$, and volume $\vec{J}$ current densities and discuss what it means to measure these quantities.

$$
\vec{J}=\rho \vec{v}
$$

A8: Magnetic vector potential due to a spinning ring of charge

Type: $\quad$ Small group

Goals: $\quad$ S2, S4, S5, S6, S7, S8, C2, C3, C4, C5

Key new ideas: Current source

Description: $\quad$ A small group activity in which students find the magnetic vector potential, $\vec{A}$, everywhere in space, due to a spinning ring of charge with radius $R$, total charge $Q$, and period $T$.

$$
\vec{A}(\vec{r})=\frac{\mu_{0}}{4 \pi} \int \frac{\vec{J}\left(\vec{r}^{\prime}\right)}{\left|\vec{r}-\vec{r}^{\prime}\right|} d \tau^{\prime}
$$


TABLE II. (Continued)

\begin{tabular}{|c|c|}
\hline \multicolumn{2}{|c|}{ A9: Magnetic field due to a spinning ring of charge } \\
\hline Type: & Small group \\
\hline Goals: & $\mathrm{S} 2, \mathrm{~S} 4, \mathrm{~S} 5, \mathrm{~S} 6, \mathrm{~S} 7, \mathrm{~S} 8, \mathrm{C} 2, \mathrm{C} 3, \mathrm{C} 4, \mathrm{C} 5$ \\
\hline Key new ideas: & Cross product in numerator, right-hand rule \\
\hline \multirow[t]{2}{*}{ Description: } & $\begin{array}{l}\text { A small group activity in which students find the magnetic field, } \vec{B} \text {, everywhere in space, due to a spinning } \\
\text { ring of charge with radius } R \text {, total charge } Q \text {, and period } T \text {. }\end{array}$ \\
\hline & $\vec{B}(\vec{r})=\frac{\mu_{0}}{4 \pi} \int \frac{\vec{J}\left(\vec{r}^{\prime}\right) \times\left(\vec{r}-\vec{r}^{\prime}\right)}{\left|\vec{r}-\vec{r}^{\prime}\right|^{3}} d \tau^{\prime}$ \\
\hline \multicolumn{2}{|c|}{ A10: Targeted homework } \\
\hline Type: & Homework \\
\hline Goals: & $\mathrm{C} 5, \mathrm{C} 6$ \\
\hline Key new ideas: & Different symmetries and/or sources \\
\hline Description: & $\begin{array}{l}\text { Problems spread throughout the sequence that ask students to find the four different fields }(V, \vec{E}, \vec{A}, \vec{B}) \text { from } \\
\text { sources with different symmetries (e.g. rectangular, cylindrical, or spherical) and/or sources that are } \\
\text { combinations of source pieces that have already been used (e.g. disks and cylinders made up of rings). }\end{array}$ \\
\hline
\end{tabular}

sequences interleaved with the ring cycle in the course. For the purposes of this paper, we have identified the ten activities in Table II as a single sequence because either students directly calculate one of four electromagnetic fields $(V, \vec{E}, \vec{A}, \vec{B})$ due to a ring-shaped source, or the activity is an essential building block for those calculations. Three other identifiable sequences are related to the ring cycle. The curvilinear coordinate sequence [61] introduces cylindrical and spherical coordinates (including inconsistencies between physicists' and mathematicians' notational conventions) and the basis vectors adapted to these coordinates $[12,17,62]$. In the integration sequence [63], students learn and/or review how to do integrals in a multivariable context, using the vector differential $d \vec{r}=$ $d x \hat{x}+d y \hat{y}+d z \hat{z}$ and its curvilinear coordinate analogues as a unifying representation $[12,17,62,64]$. The content contained in these two sequences is a necessary prerequisite to the ring cycle. In the (optional) power series sequence [65], students learn and/or review how to do power series using concepts, terminology, and techniques that are common among physicists, but not often taught in mathematics courses.

Keeping track of how prerequisite knowledge from various interleaved sequences needs to build over time can be a daunting prospect. The Paradigms team is building interactive online learning progressions that encode what we have learned about sequencing content [66].

\section{METHODS AND SETTINGS}

\section{A. Data and analysis}

In order to identify the tactics that were used to develop the ring cycle, we used an analytical approach similar to that of collaborative autoethnography [67]. Following in this tradition, our analysis was self-focused, in that we are each functioning as both researcher and participant; researcher visible, in that our goal is to make more visible our thoughts, processes, and commitments during curriculum development; context conscious, in that we are grounding our experiences in our particular local environments; critically dialogic, in that there is an ongoing dialogue between our roles as researcher and participant; and interactive, in that our analysis of self is enhanced by the questioning and probing of the other.

We selected the ring cycle for analysis because we have each used it several times in our own classrooms and adapted it to our local contexts. Our data consisted primarily of: public records of the ring cycle activities [9]; research articles detailing aspects of the Paradigms in Physics project $[10,11,23,29]$ and the ring cycle activities [12-14,1622,24-26]; archival written notes, emails, and reflections during the original development and various implementations and adaptations; personal memories; and finally, ongoing individual and collaborative reflections about our development and adaptation processes. Our analysis involved individual reflection and writing interspersed with collaborative meetings in which we probed each other's memories and experiences and worked to synthesize and interpret our data and articulate the patterns uncovered, proceeding iteratively until we reached consensus.

Early reflections and discussions primarily focused on generating rich descriptions of our local environments (see Sec. IV B) and our own implementations of the ring cycle, particularly attending to why we made the choices we made and any changes that were made over time. Analysis and interpretation at this early stage focused on identifying, articulating, and synthesizing the higher-level theoretical perspectives and research basis that had informed our development and adaptation (see Sec. II B). We then checked (and confirmed) that all three implementations 
TABLE III. A comparison of the features of each institution.

\begin{tabular}{|c|c|c|c|}
\hline Feature & OSU Paradigms & CSU San Marcos & DePaul \\
\hline Electromagnetism I & Two 3 week Paradigms & 15 week semester & 10 week quarter \\
\hline Class time/week & $370 \min$ & $150 \mathrm{~min}$ & $180 \min$ \\
\hline Class meetings/week & 5 & 2 & 2 \\
\hline Total class time & $37 \mathrm{~h}$ & $37.5 \mathrm{~h}$ & $30 \mathrm{~h}$ \\
\hline Math background & $\begin{array}{l}\text { Most have had vector } \\
\text { calculus }\end{array}$ & $\begin{array}{l}\text { Most have had math methods } \\
\text { and vector calculus }\end{array}$ & $\begin{array}{l}\text { Math methods required, most } \\
\text { had vector calculus }\end{array}$ \\
\hline Physics background & juniors & juniors and seniors & $\begin{array}{l}\text { juniors and seniors (offered } \\
\text { every other year) }\end{array}$ \\
\hline Electromagnetism II & 10 week quarter & 15 week semester & 10 week quarter (not required) \\
\hline
\end{tabular}

were consistent with this synthesis and the focus shifted to articulating how the these higher-level principles were instantiated in the ring cycle.

During the analysis, and partly in response to reviewers of an earlier version of this manuscript, it became clear that we had each been using a number of "guidelines" that were not as high-level as design principles, a level that we found to be missing in discussions in the literature. Thus, our later analysis focused on explicitly articulating what we are now calling design tactics. We reanalyzed our data in order to identify the tactics that we had been using in the original development and in our various adaptations. The resulting set of design tactics (presented in Sec. V) represent a combination of tactics we have been using explicitly for years (often shared over time as a part of our collaborations) and those we have been using implicitly that were uncovered or articulated through our analysis.

\section{B. Institutional contexts}

The three sites included in this analysis span a range of settings. In this section, we briefly describe these settings. Table III provides an overview of the format and structure of the upper division electromagnetism courses at each site.

It is important to note that in addition to being physics education researchers, each of the authors was allowed considerable autonomy in designing the course at the their institution, has colleagues who support their approach, and has taught the course multiple times, providing continuity, expertise, and iterative adjustments.

\section{Original implementation: $O S U$}

OSU is a typical medium-sized, public R1 research university in the Pacific Northwest. The physics department offers the entire complement of undergraduate and graduate degrees with a variety of options. In 1997, C. A. M. was the developer of the Static Fields and Symmetries and Idealizations courses in the initial implementation of the Paradigms redesign. She was given freedom to develop activities and the sequence of content in the courses, subject to the constraint that it cover the material from Chaps. 1, 2, and 5 of Griffiths's electromagnetism textbook [59]. She worked collaboratively with the entire Paradigms team to fit this course within the program, particularly paying attention to sequencing with a vector calculus course being simultaneously developed in the mathematics department as part of the Vector Calculus Bridge Project [62] and with the senior-level electromagnetism capstone course which covers more advanced electromagnetism topics. C. A. M. has continued to teach these two Paradigms frequently since 1997.

\section{Secondary implementation 1: CSUSM}

California State University San Marcos (CSUSM) is a diverse, public, regional comprehensive university in Southern California. The physics department offers a bachelors degree in applied physics (there is no graduate program). The degree program was launched in 2008, and upper division electromagnetism was first offered in that year. Developing a new course, in a new program, provided an opportunity to take a nontraditional approach. Seeking materials to actively engage students in class, and being aware of the Paradigms project, E. P. consulted with C. A. M. and the Paradigms website to learn more about the electromagnetism materials and the ring cycle specifically. In spring 2009, E. P. incorporated most of the material from the two, three-week Paradigms courses, including the ring cycle. Discussions with C. A. M. during the semester before and during this semester helped E. P. develop a deeper understanding of the learning goals, design principles, and practical implementation of the Paradigms materials. He has continued to use the materials, and has served as a consultant on several OSU-based projects involving the Paradigms.

\section{Secondary implementation 2: DePaul}

DePaul University is a large, diverse, private, Catholic university. The physics department offers both bachelor's and master's degrees in physics. Electromagnetism is a two-quarter sequence offered in alternating years and thus includes both juniors and seniors (many of whom have 
TABLE IV. A summary of the design tactics identified through analysis of the ring cycle. The sections indicate where each tactic is first introduced, but are not intended to imply that this is the only context to which that tactic applies.

Content design tactics (Sec. VA)

DT1-Task analysis: Perform a fine-grained task analysis to divide a task into constituent elements.

DT2 - Build complexity: Give students experience with the basic "building blocks" and add elements and complexity with activity sequences.

DT3 - Challenge level: Adjust the level of difficulty to stay squarely within the zone of proximal development for the majority of students.

DT4-Iterative development: Use formative assessment across multiple iterations to refine the design of activities, with special attention to sequences, such as adding activities where needed.

Sensemaking design tactics (Sec. V B)

DT5-Problem-solving choices: Make prompts open-ended and/or underspecified to provide opportunities for professional-level problem-solving choices.

DT6-Representations: Create prompts that expect students to generate and translate between representations.

DT7-Generality: Frame special cases as limits of general cases.

Classroom practice design tactics (Sec. V C)

DT8-Alignment: Match the demands of different activities with the pedagogical affordances of different class components (e.g., small group activity, kinesthetic activity, lecture, homework).

DT9-Interaction: Craft interactions among students, instructors, and tools to support the task learning goals.

DT10-Classroom norms: Establish norms that emphasize that everyone contributes to scientific reasoning and sometimes gets things wrong.

already taken classical and/or quantum mechanics). In addition, both quarters are not required for all majors.

As a postdoc at OSU, M. B. K. co-taught (with C. A. M.) both electromagnetism Paradigms and taught on her own the first of the electromagnetism Paradigms. This experience allowed her to experience the ring cycle as an observer and instructor. Working and co-teaching with C. A. M. also allowed her to become familiar with the theoretical perspectives foundational to the Paradigms curriculum (see Sec. II B) [68].

When joining the DePaul faculty, M. B. K. reorganized the electromagnetism course sequence to account for the fact that not all students take both quarters. In the new structure, the first quarter focuses on electro- and magnetostatics in vacuum (Griffiths [59] Chaps. 1, 2, 5, and parts of 3) and moves solutions methods (Chap. 3), fields in matter (Chaps. 4 and 6), and electrodynamics (Chap. 7) to the second quarter, covering more advanced topics as time allows. With this reorganization, M. B. K. also chose to incorporate many of the Paradigms activities and sequences into the first quarter, including the ring cycle.

\section{DESIGN TACTICS}

In the following subsections, we describe the design tactics used in developing the ring cycle and provide examples for each to illustrate how they relate to the process of curriculum development. These design tactics are summarized in Table IV. We are presenting the design tactics in three groups: those related to content (Sec. VA), sensemaking (Sec. VB), and classroom practice (Sec. V C). However, this grouping is not intended to imply that the design tactics are used only in these areas; rather, design tactics are presented in the area that provides a clear example of their use. Following the introduction of the design tactics, we discuss how these design tactics were used in adapting the ring cycle in the secondary implementations (Sec. V D).

\section{A. Content design tactics}

The first four design tactics are framed around applying general principles or research findings in order to accomplish content learning goals: how to divide the learning tasks into constituent pieces, sequence the pieces in increasing level of difficulty, refine tasks to stay within the zone of proximal development of most students, and use iterative development and formative assessment to refine the activities as needed.

\section{Design tactic 1-Task analysis: Perform a fine-grained task analysis to divide a task into constituent elements}

The centerpiece of the ring cycle is a set of four activities (A5, A6, A8, A9) in which groups of students are asked to find an electromagnetic field due to a (static or spinning) ring of charge. In the first ring activity, A5, students generalize the expression for the potential due to a point charge to a ring of charge:

$$
V=\frac{k Q}{r} \rightarrow V(\vec{r})=\int \frac{k \lambda\left(\vec{r}^{\prime}\right)\left|\vec{d} r^{\prime}\right|}{\left|\vec{r}-\vec{r}^{\prime}\right|} .
$$

The formula for the electrostatic potential $V(\vec{r})$ in this case involves several elements: linear charge density $\lambda$, the 
infinitesimal length element $\left|d \vec{r}^{\prime}\right|$, and the distance formula $\left|\vec{r}-\vec{r}^{\prime}\right|$, which students must recognize and be able to write in an appropriate coordinate system.

In order to accomplish the task, one must first break the problem up into manageable pieces:

- understand the geometry of the problem, typically by drawing a figure,

- find a expression for the linear charge density,

- chop up the source into "infinitesimal pieces"

- write a coordinate dependent expression for the infinitesimal length element,

- recognize that they must calculate the potential at the point where they are trying to evaluate it due to each of the chopped up pieces that live elsewhere in space,

- understand how to coordinatize the denominator,

- set up the correct integral

- include appropriate limits of integration.

Each of these many actions addresses one of the content learning goals and many are explicitly addressed with one of the preceding activities (such as how to write the distance formula in curvilinear coordinates, A3). It is valuable for the instructional team [69] to keep this long list of actions in mind as different groups stumble on different elements.

\section{Design tactic 2-Build complexity: Give students experience with the basic building blocks and add elements and complexity with activity sequences}

The other three ring activities (A6, A8, and A9) follow the same pattern as the ring activity for $V$. The elements of the calculation for $V$ in A5 also appear in the subsequent calculations of $\vec{E}, \vec{A}$, and $\vec{B}$. Yet these later calculations require additional elements as well. The activities are arranged so that the calculational complexity increases from one activity to the next. For example, the most complicated field, the Biot-Savart law for the magnetic field $\vec{B}$ (A9), involves a current density (a charge density times a velocity), a cross product, and a vector-valued differential $d \vec{r}$.

\section{Design tactic 3-Challenge level: Adjust the level of difficulty to stay squarely within the zone of proximal development for the majority of students}

Early in the development of the ring cycle, we found that students did not have enough experience with charge or current densities to be able to complete the ring activities. Although they were somewhat comfortable with constant volume mass densities, they had little opportunity to solve problems with nonconstant, two- or three-dimensional, charge and current densities. This result motivated the development of the kinesthetic activities (A4 A7) where students act out (linear, surface, volume) charge and current densities with their bodies.
Design tactic 4-Iterative development: Use formative assessment across multiple iterations to refine the design of activities, with special attention to sequences, such as adding activities where needed

There are two major ways in which we have altered the ring cycle over time based on formative assessment, including responses of students to in-class activities, homework, and exams. Both approaches particularly attend to sequencing and thus represent a specific focus for our iterative development. The first, in which tasks are added as needed, was described in the previous design tactic. In the second, the content of the tasks is changed.

The decision to use a similar physical context (a ring) for activities exploring different fields was made in response to lessons learned during the first few years of the development of the ring cycle. Initially, C. A. M. made the choice to present a sequence of activities that covered all of the coordinate systems (rectangular, cylindrical, and spherical) and all of the fields $(V, \vec{E}, \vec{A}, \vec{B})$ by pairing each field with a source that was symmetric with respect to a different coordinate system. A number of groups struggled on the in-class activities because pairing different coordinate systems with different fields did not give enough practice with either for many students to master anything. This difficulty motivated a shift to the current activities, all employing the same geometry so that student groups can focus on the physical and calculational differences among the various fields. Practice with other geometries is provided in homework (A10), when students have more time to coordinate the additional algebraic difficulty.

\section{B. Sensemaking design tactics}

The next three design tactics focus on ensuring that students have the opportunity (and even the need) to carry out common professional practices to address particular sensemaking learning goals. In this section in particular, we make no claim that this set of design tactics is complete. We have merely chosen to illustrate a few of many possible sensemaking design tactics that are well illustrated by the ring cycle: providing students the opportunity to make appropriate problem-solving choices; to generate representations and translate from one representation to another; and to use symmetry as a way to explore special cases of a more general expression.

\section{Design tactic 5-Problem-solving choices: Make prompts open ended and/or underspecified to provide opportunities for professional-level problem-solving choices}

A first simple example: In the four primary ring activities (A5, A6, A8, A9), students are given the iconic formula listed in Table II for each of the fields in terms of a volume source density, rather than the formula for linear charge and current densities required by the specific example. This 
choice creates a need for student groups to talk through the different types of sources and chose a dimensionally appropriate version of the formula.

A deeper second example: The $\vec{E}$ ring activity (A6) is the first of the ring activities where the integrand is a vector field. In the final steps of this problem, it is necessary to use curvilinear coordinates (to make the denominator easier) but rectangular basis vectors (to make integration possible) - a highly nontrivial set of professional problemsolving choices. Most student groups stumble on this point. (Because the curvilinear basis vectors change from point to point in space, it is impossible to compute the integral using curvilinear basis vectors.) Sequencing the $\vec{E}$ ring activity after the $V$ ring activity enables groups to focus their attention on this point alone in the second activity. With help, they are able to conclude that when they use rectangular basis vectors the integral of a vector field decomposes into three separate integrals with unit vectors attached.

\section{Design tactic 6-Representations: Create prompts that expect students to generate and translate between representations}

Figures, especially figures that encode geometric relationships are a representation that students must not only use to translate from a physical situation to an algebraic representation, but also be able to generate and elaborate at need. The prompt for the four ring activities is given from a hula hoop rather than a drawing. "This hula hoop represents a ring of charge. The radius of the ring is $R$ and the total charge is $Q$. Calculate the electrostatic potential $V$ at an arbitrary point in space." (Later magnetic field activities have similar prompts which include a period $T$ for the spinning ring.) By not providing a figure in the problem statement, students must draw and appropriately label their own figure, including adding the two position vectors $\vec{r}$ and $\vec{r}^{\prime}$ when needed.

\section{Design tactic 7-Generality: Frame special cases as limits of general cases}

The primary four ring activities (A5, A6, A8, A9) ask students to find the relevant field at an arbitrary point in space rather than on the axis of symmetry. Follow-up prompts in class ask them to evaluate their expression on axis or in the plane of the ring. This order provides several important pedagogical affordances: Students recognize that the distance between source and field point is not constant and cannot be factored out of the integral. Students also experience directly that not all integrals can be computed analytically, in closed form. Finally, students are not led to believe that only problems with high symmetry can be solved. Instead, symmetric problems are situated as an opportunity for limiting case analysis [70,71].

\section{Classroom practice design tactics}

The final three design tactics are framed around classroom practice: placing activities in the course so that they align with the available learning resources, choosing pedagogical strategies appropriate to the task, and setting classroom norms, especially ensuring that all students have the opportunity to participate in all of the professional practices, such as contributing to scientific reasoning and discourse.

\section{Design tactic 8-Alignment: Match the demands of different activities with the pedagogical affordances of different class components}

The difficulty and newness of the primary four ring activities (A5, A6, A8, and A9) suggests the benefit of intensive working with peers and the teaching team. Hence, in the ring cycle, these tasks are done as in-class, group problem-solving activities.

Kinesthetic activities, such as the charge density (A4) and current density (A7) activities involve a number of students in interaction with the instructional team. Obviously, an in-class setting is required. Furthermore, as kinesthetic activities naturally engage students' embodied cognition, these activities are great for exploring geometric relationships. Because students respond in quick succession, to prompts like: "Make a linear charge density," "make a nonconstant linear charge density," "make a surface charge density," etc., these activities are also conducive to addressing technical vocabulary.

In the current density activity, most students do not know the how to describe the relationship between charge density $\rho$, velocity of the charges $\vec{v}$, and current density $\vec{J}$ :

$$
\vec{J}=\rho \vec{v},
$$

nor how to use this relationship to "measure" the current by counting the number of charges that go through a twodimensional "gate" in one second. Thus, this content is best handled by a mini lecture within the overall current density activity and can be supported by appropriate reading.

In contrast, other tasks may require different pedagogical resources. When students must express the distance formula in the denominator of the ring problems,

$$
\left|\vec{r}-\vec{r}^{\prime}\right|=\sqrt{\left(x-x^{\prime}\right)^{2}+\left(y-y^{\prime}\right)^{2}+\left(z-z^{\prime}\right)^{2}},
$$

in cylindrical coordinates

$$
\left|\vec{r}-\vec{r}^{\prime}\right|=\sqrt{s^{2}+s^{\prime 2}-2 s s^{\prime} \cos \left(\phi-\phi^{\prime}\right)+\left(z-z^{\prime}\right)^{2}} .
$$

The transformation of coordinates relies heavily on students' individual facility with trigonometric identities but typically does not require as much support from the 
instructional team. Hence, in the ring cycle, this task is assigned as a homework problem prior to doing the first ring activity (A5). Students can spend as much or as little time on it as they personally need and are then prepared to contribute to the relevant conversations during the in-class activity.

\section{Design tactic 9-Interaction: Craft the form of interactions among students, instructional staff, and tools to support the task learning goals}

Applying DT8 (alignment) allows us to choose which activities should be done in small groups in class. For these activities to be effective, it is necessary for the students to have individual space to record and refine their own ideas and to be able to see each others' work in order to collaborate. It is also necessary for the instructional team to monitor the work of many groups and choose when to intervene. Therefore, we choose to have students work in groups of 3 , using a $2^{\prime} \times 3^{\prime}$ whiteboard placed on a table as a shared brainstorming space. The work on the whiteboard is easily visible to the teaching team and easily available for them to point at and write on, as needed.

In a similar way, while many students are able to reason about the geometric relationships in the ring directly from a figure sketched on their whiteboards, there are often a few students who have trouble with understanding such figures as representing three-dimensional relationships. It can be helpful to provide these latter students with the hula hoop (described in DT6) so that they can handle, point to, and even write on it.

\section{Design tactic 10-Classroom norms: Establish norms that emphasize that everyone contributes to scientific reasoning and sometimes gets things wrong}

After several years of teaching the ring cycle, author C. A. M. noticed that many students were unfamiliar with both the common professional notation for the position vector $\vec{r}$ and its relationship to the origin and to the distance formula. Enter the Star Trek activity (A1). This activity is framed as an in-class role-playing skit based on characters in the science fiction TV show and movie series by that name. The instructor begins by explaining the premise of the play, especially for students who may be unfamiliar with the show or characters. Two characters: Captain Kirk and Mr. Spock, are separated while exploring a planet. The chief engineer, Scotty, who is on their starship, must "beam" (teleport) Mr. Spock to Captain Kirk's location to save him from an attack by aliens. To do this, the rest of the starship crew must figure out the distance between Kirk and Spock. The instructor plays Scotty, two students volunteer to play the role of Kirk and Spock, and the remaining students play the role of crew members and take turns coming to the board to outline a solution to the problem. To find the appropriate distance, Spock and Kirk must both describe their position vector relative to an origin - a large red building that is fortunately visible to all protagonists. In this activity, both the concept of and a notation for the position vector $\vec{r}$ are established and the role of the origin is highlighted. The distance in rectangular coordinates [Eq. (3)] is calculated collaboratively by the crew in consultation with Scotty, who promptly promotes the successful red shirt crew members. (Note that in the original TV show, crew members with red shirts often died by the end of the episode, so promotion is a very good thing.)

At OSU, the ring cycle occurs right at the beginning of the first middle-division course, so C. A. M. uses the Star Trek activity to set several classroom norms. The instructor stands on a table during the play (because she is on the Starship Enterprise in orbit above the planet). This act establishes both that this classroom will not be like any other that the students have experienced and that, if she can be silly enough to do this, then perhaps it is safe for the students to take intellectual risks as well. Also, since she is stuck on the table, the other crew members must come to the board to sketch out solutions, establishing that the roles of teacher and learner are fluid. Typically, it requires input from several crew members before the distance formula has been correctly formulated, establishing that different people may have different elements of a correct solution so that collaboration is useful.

We consider all of these classroom norms to be essential in facilitating productive interactions, although, of course, in different institutions these norms will be established at different times and in different ways. For example, at DePaul, the Star Trek activity (A1) is implemented differently in ways both small and large. This is described next in Sec. V D.

\section{Secondary implementations}

As described in Sec. II C, instructors adopting instructional methods and materials often make modifications. Dancy and Henderson [52] point out that some degree of modification is likely to be necessary because of differences in instructor preference and local circumstances. The circumstances requiring customization may include differences in student population and preparation, local program design (e.g., which topics are covered in which courses), and course format. Consistent with this finding, M. B. K. and E. P. made modifications to the ring cycle when they used it at their institutions. Both institutions differ significantly from the original development site (as discussed in Sec. IV B) and have more typical electromagnetism courses that are part of more standard physics degree programs (as compared to the Paradigms). In this section, we provide examples of how making these adaptations can be understood as the reapplication of the design tactics given awareness of the new context.

Table III shows the format and structure of the upper division electromagnetism courses at OSU, CSUSM, and 
DePaul. At OSU, two, three-week Paradigms courses cover electro- and magnetostatics (with other topics covered in a one quarter-long capstone) [72]. These topics are covered in the first of two semester-long courses at CSUSM and the first of two quarter-long courses at DePaul. The ring cycle is used in the first electromagnetism course at all three sites.

At CSUSM, the ring cycle is used in a course with less overall class time, and shorter, less frequent class meetings. To accommodate this, E. P. has made some adaptations in individual activities. One example is in the $V$ for 2 charges activity (A2), where students are asked to calculate an exact and approximate potential for two point charges in a variety of cases (identical charges or dipole, and in different limiting geometries). At CSUSM, all student groups work through the first case in class, ending with a brief discussion of the key points in the calculation. Students are assigned one or more of the remaining cases as homework for the next class, which begins with groups conferring and putting their solution on a whiteboard. Groups present their work to the class, leading to a discussion that compares and contrasts the cases. This approach reduces the class time needed for the activity by shifting some of the calculation to homework, but only after students have successfully solved one case working with other students and with the instructor's support.

Breaking the $V$ for 2 charges activity (A2) across in- and out-of-class time is an example of using DT8 (alignment) to adapt an activity to a local setting by aligning tasks and course components. Students first attempt the calculation with support from the instructor and other students. They then work on variations as homework, when they (generally) have less support but also, because of the in-class work, need less. Students can also take the time to work through and check the details of the calculation; the sustained focus this requires may be better suited to homework than a noisy classroom environment. Finally, students can check their work, confirm their understanding, and resolve lingering issues during the second round of group work and whole class discussion, taking advantage of the resources available during in-class time.

In the secondary implementation at DePaul, M. B. K. made some changes in the Star Trek activity (A1), where students "derive" the Cartesian distance formula, in order to highlight different classroom norms (DT10) than those at OSU. At OSU, the emphasis in this activity is on establishing an environment where the students feel safe being publicly wrong, so that students are encouraged to interact with others moving forward. This is best done by instructor modeling in a whole class discussion. However, at DePaul, students come into the upper-division courses with the expectation of interacting with each other regularly since all of their previous physics courses are taught in a studio environment and use active engagement approaches. Therefore, a more pressing need is the tendency of a mixed class of juniors and seniors to separate and M. B. K. instead chooses to have them work in small groups that intentionally mix cohorts in order to facilitate future interactions.

Another small change in this activity related to classroom norms (DT10) is that M. B. K. uses the characters of Kirk and Uhura (one of the few female characters in the original show) instead of Kirk and Spock. Like other physics departments, upper-level courses at DePaul tend to be majority male (for the three years that M. B. K. has taught the class, women and gender minorities comprised $17 \%(4 / 23), 35 \%(6 / 17)$, and $30 \%(3 / 10)$ of the class). This small change was done to provide an added way for the women in the class to connect to the story.

\section{DISCUSSION}

In this section, we provide some synthesis and discussion of the design tactics introduced in Sec. V. The sections mirror those in Sec. V, but highlight the fact that these design tactics are not limited to the category in which they were introduced. These sections explore how the design tactics interact with each other as well as how they are related to and can inform conversations about curriculum development in the larger community. The last section provides some synthesis and discussion around the application of these design tactics in adapting curricular materials in other contexts.

\section{A. Discussion of content design tactics}

While each of the design tactics presented in Sec. VA is grounded in the research and theoretical perspectives outlined in Sec. II B, these tactics provide a tighter connection to how activities within the ring cycle accomplish the content learning goals shown in Table I. First, the use of a task analysis identifies the building blocks for a complex task (DT1), which are then sequenced in a logical progression that builds these elements together by gradually adding complexity (DT2). Then, this sequence is turned into activities at a appropriate level such that the majority of students are working on tasks that they may not be able to do on their own, but can do with appropriate guidance (DT3). This refinement is conducted in response to ongoing formative assessment both within a given single course as well as across multiple iterations of the sequence (DT4).

It is important to note that these tactics are not orthogonal, but intersect and interact with each other. For example, sequencing which builds complexity (DT2) can be done on multiple levels. The task analysis used to demonstrate DT1 (identifying the elements needed to find the potential due to a charged ring), led to a sequencing of activities (A1-A5) that is as much of an illustration of building complexity, albeit at a smaller scale, as the sequencing of the four different fields (A5, A6, A8, A9).

While these four tactics highlight how the curriculum development process addresses the content learning goals, 
DT3 (challenge level) and DT4 (iterative development) are much broader in scope than just addressing content. In fact, these overarching tactics can and should be used throughout the development process, as well as when adapting to local context, as discussed in the following sections. Of course, when a curriculum is "finished" and disseminated as final product, continuing iterative improvement may end. This tension is a challenge for curriculum developers. Disseminating materials in editable form can help resolve this, and should be an on-going conversation within our community.

\section{B. Discussion of sensemaking design tactics}

The three design tactics identified in Sec. V B begin to articulate a set of strategies that can provide students with a broad set of opportunities to think like a physicist. These tactics are directly relevant to the ring cycle and are designed to connect the research basis discussed in Sec. II B with the sensemaking learning goals of the course (see Table I). For courses with goals that operationalize thinking like a physicist differently, we expect that different design tactics would and should be used.

As mentioned in Sec. VIA, creating activities that maintain an appropriate level of challenge for most students (DT3) is broader than just addressing content goals and it is also important to consider this tactic in conjuction with the design tactics discussed in this section. For example, when using open-ended and ambiguous prompts to provide opportunities for professional-level problemsolving choices (DT5), the timing of intervention is critical. Students need the chance to do what they can, make choices, get stuck, try to get themselves unstuck, and generate questions. At this point, students are at the point where guidance is appropriate. Understanding when and how to intervene in order to appropriately hit the zone of proximal development for students is also related to our last set of design tactics, which are focused on larger classroom practice issues.

\section{Discussion of classroom practice design tactics}

The design tactics identified in Sec. VC focus on the types of activities that will facilitate interactions that will support students in learning both content and how to think like a physicist. They also highlight how to create settings where these interactions can happen and how to ensure that the appropriate resources are available to students as they undertake these tasks.

Just as DT3 (challenge level) and DT4 (iterative development) are broader in scope than just addressing content issues, we also view DT8 (alignment) as a tactic that is foundational to all design decisions and one that is not explicitly discussed as such in the literature on curriculum development.

Many excellent sets of curricular materials (e.g., Tutorials [73], Peer Instruction [74], RealTime Physics
[75]) employ a single pedagogical approach, typically because they are intended to be used in a restricted pedagogical setting (recitation, large lecture, and lab, respectively). In smaller classes without these constraints and with more complex upper-division content, we have found it both possible and advantageous to use a mixture of pedagogical approaches. In this setting, DT8 (alignment) becomes particularly important.

There are many components to a course (different types of in-class activities, homework, office hours, exams, etc.) that each have particular affordances. For example, during class when students and the instructor are all in the same place at the same time, students can interact with each other and with the instructor. Help and feedback is quickly available, the instructor can monitor students' progress (i.e., formative assessment made possible by access to evidence of student thinking), and address the entire class if needed. In contrast, when students work outside of class (such as on homework), they may have access to other students, textbooks, and online materials, but limited access to the instructor. However, students can generally take more time with homework, stopping and starting, returning to a problem after seeking help or simply taking a break.

Just as a course has many components, we also expect students to engage in several different types of tasks, just as professional physicists do. [Note that the intent of DT1 (task analysis) is to help the development team unpack and identify these tasks.] For example, when solving a complex problem, we expect students to be able to analyze the problem conceptually, diagram the situation, work through detailed calculations, explain their reasoning and choices, and reflect on the result. In addition, students are also learning to perform new procedures; apply techniques in different situations; use, generate, and translate between different representations; and many other kinds of tasks. Thus, DT8 (alignment), is about achieving the best match between the demands of tasks and the affordances of the different class components.

The process of alignment is intimately tied to the other classroom practice design tactics (see Sec. V B). Alignment (DT8) weighs the question of what types of interactions are best supported in each context. Once a choice is made, one can craft the activity to take advantage of the affordances of the context in order to facilitate interactions that will support learning (DT9). Finally, one must establish appropriate classroom norms (DT10) in order to ensure that these interactions support equitable participation.

One example of how these tactics interplay is in the design of what is assigned for homework. It is not unusual for faculty to design their courses such that challenging problems are assigned to homework, with the expectation that if students need help, they will come to office hours. However, this expectation does not attend to some of the potential inequities around students' lives outside of class (e.g., students who work and cannot attend office hours) 
and these inequities may be magnified depending on the type of institution. The intersection of the three classroom practice design tactics (DT8, DT9, DT10) means that we want students to get stuck on difficult points where there are other people around to help. Thus, we move challenging problems, such as those that require getting the conceptual feel of a problem, explaining one's reasoning, or justifying one's choices, into the classroom where everyone can benefit from the formative feedback of working with peers and the instructor. In contrast, students have more time but less access to feedback during homework, so we use homework for tasks that are more time consuming, but that are routine, provide practice, or apply and extend work done in class to other contexts (e.g., A10 and E. P.'s adaption of A2 discussed in Sec. V D).

Finally, two broader points of discussion that apply to all three types of design tactics. First, we note that there are tensions among some of the design tactics. Sometimes one must decide which tactics to emphasize in a particular situation. Making the tactics explicit facilitates resolving these tensions and helps make the design process explicit as well. For instance, a task analysis (DT1) can reveal many constituent elements of a task and there can be an efficiency to leading students through these elements; but giving students opportunity to make professional-level problemsolving choices (DT5) may require allowing them to choose their own path through a problem solution. An example of this occurs in the ring cycle when students calculate the electrostatic potential due to a charged ring (A5). The task analysis reveals a large number of pieces to this problem (see Sec. VA). The prompt to students, however, is a hula hoop (rather than a drawing), and parameters for the ring's radius and total charge. While one could enumerate individual steps for students, the prompt requires that they identify and sequence the necessary steps themselves. Learning goals can help weigh the different, competing priorities. In this example, emphasis is placed on providing opportunity for students to make professional choices (learning goal S1), rather than identifying and sequencing the steps in this problem for students. Exploration of tensions within these design tactics and the consequences of potential trade-offs is an area for further work.

Second, it is important to note that the design tactics do not stand on their own. They stem from and are steeped in the broader research and theoretical perspectives outlined in Sec. II B. In particular, DT8 (alignment) relies heavily on understanding both the cognitive demands of tasks (as outlined in the design tactics discussed in Sec. VA) as well as the sociocultural aspects of the class components and a commitment to promoting equitable classroom participation.

\section{Discussion of secondary implementations}

When adopting curricular materials or instructional practices, adaptions are often needed to address the local setting and context. The changes described in Sec. V D are examples of such adaptations. Although the design tactics were not formulated in advance and then applied to make these changes, our retrospective analysis shows that the changes made were consistent with application of the design tactics. That is, the same design tactics, applied in a different context (instructional format, student population, course or program goals), can lead to a different version of an activity. Based on this, we suggest that prospective users of a curriculum can intentionally use that curriculum's design tactics to guide productive or necessary adaptations that will be consistent with the original development. Of course, this assumes that the original developers have made the design tactics explicit and public, which is something that is not currently done in the literature.

Clearly, adopters may wish to make changes outside the scope of the design tactics. This could include using different or additional design tactics. However, if adopters are considering changes that are at odds with the original design principles, we suggest that the materials may not be a good choice. This is not to say that an adopter cannot glean something useful from a curriculum in such a case. However, changes at the level of design principles are more likely to be modifications of what Henderson and Dancy [52] call "essential components."

\section{CONCLUSIONS}

This article has proposed design tactics as a tool for curriculum development, and presented ten tactics identified from a retrospective analysis of the development of the ring cycle. Design tactics are intended to be more concrete and guide the development of activities more directly and with greater specificity than design principles. While the particular ring cycle design tactics may be useful to others developing similar materials, we hope these examples of design tactics will be useful for curriculum developers interested in creating their own design tactics. We expect this will make their work more systematic and consistent. We also anticipate that the articulation of design tactics may provide a fruitful mechanism for comparing curricula.

Design tactics can also be useful tools for curriculum adopters. We have described two secondary implementations of the ring cycle, and how adaptations for these new contexts are consistent with the (re)application of the ring cycle design tactics. Based on this, we suggest that knowledge of a curriculum's design principles and design tactics can be useful for potential adopters. We therefore encourage developers to make their principles and tactics explicit and public.

\section{ACKNOWLEDGMENTS}

This paper was supported in part by NSF Grants No. 1323800 and No. 1836603. Much of the development 
of the ring cycle was supported by earlier NSF grants to the Vector Calculus Bridge Project No. 0088901 and No. 0321032 and to the Paradigms Project, especially $9653250,0618877,1023120$. C. A. M. would like to thank the whole Paradigms team, faculty, postdocs, TAs, LAs, and especially students, whose collective insights have been essential to this work. Special acknowledgement goes to Tevian Dray, PI on the Vector Calculus Bridge Project, Elizabeth Gire for her role in the collaboration between C. A. M. and E. P., and feedback from the OSUPER group.
[1] Physport, https://www.physport.org/, accessed: 2019/ $10 / 18$.

[2] L. R. Beach, Image Theory: Theoretical and Empirical Foundations (Routledge, London, 1998).

[3] L. C. McDermott, Research as a guide for curriculum development: An example from introductory electricity. Part I: Investigation of student understanding, Am. J. Phys. 60, 994 (1992).

[4] P. S. Shaffer and L. C. McDermott, Research as a guide for curriculum development: An example from introductory electricity. Part II: Design of instructional strategies, Am. J. Phys. 60, 1003 (1992).

[5] F. Goldberg, V. Otero, and S. Robinson, Design principles for effective physics instruction: A case from physics and everyday thinking, Am. J. Phys. 78, 1265 (2010).

[6] F. Goldberg, E. Price, S. Robinson, D. Boyd-Harlow, and M. McKean, Developing the learning physical science curriculum: Adapting a small enrollment, laboratory and discussion based physical science course for large enrollments, Phys. Rev. ST Phys. Educ. Res. 8, 010121 (2012).

[7] S. V. Chasteen, K. K. Perkins, P. D. Beale, S. J. Pollock, and C. E. Wieman, A thoughtful approach to instruction: Course transformation for the rest of us, J. Coll. Sci. Teach. 40, 24 (2011).

[8] G. Morrison, S. Ross, J. Morrison, and H. Kalman, Designing Effective Instruction (Wiley, New York, 2019), ISBN 9781119465935.

[9] Paradigms in Physics, https://paradigms.oregonstate.edu, accessed: 2020/01/30.

[10] C. A. Manogue, P. J. Siemens, J. Tate, K. Browne, M. L. Niess, and A.J. Wolfer, Paradigms in Physics: A new upper-division curriculum, Am. J. Phys. 69, 978 (2001).

[11] C. A. Manogue and K. S. Krane, Paradigms in Physics: Restructuring the upper, Phys. Today 56, No. 9, 53 (2003).

[12] T. Dray and C. A. Manogue, The vector calculus gap: Mathematics $\neq$ Physics, PRIMUS 9, 21 (1999).

[13] K. S. Meyer, Masters thesis, Oregon State University, 1998.

[14] K. P. Browne, Ph.D. thesis, Oregon State University, 2001.

[15] C. A. Manogue, K. Browne, T. Dray, and B. Edwards, Why is Ampère's law so hard? A look at middle-division physics, Am. J. Phys. 74, 344 (2006).

[16] L. T. Cerny, Masters thesis, Oregon State University, 2007.

[17] T. Dray and C. A. Manogue, Putting differentials back into calculus, College Math. J. 41, 90 (2010).

[18] C. A. Manogue, L. Cerny, E. Gire, D. B. Mountcastle, E. Price, and E. H. Van Zee, Upper-division activities that foster "Thinking Like A Physicist", AIP Conf. Proc. 1289, 37 (2010).
[19] E. van Zee and C. Manogue, Documenting and interpreting ways to engage students in 'Thinking Like a Physicist', AIP Conf. Proc. 1289, 61 (2010).

[20] L. T. Cerny, Ph.D. thesis, Oregon State University, 2012.

[21] E. Gire and E. Price, Graphical representations of vector functions in upper-division E\&M, AIP Conf. Proc. 1413, 27 (2012).

[22] E. Gire and E. Price, Arrows as anchors: Conceptual blending and student use of electric field vector arrows, AIP Conf. Proc. 1513, 150 (2013).

[23] E. Gire, M. B. Kustusch, and C. Manogue, Supporting and sustaining the holistic development of students into practicing physicists, AIP Conf. Proc. 1513, 19 (2013).

[24] J. P. Zwolak, M. B. Kustusch, and C. A. Manogue, Rethinking the rubric for grading the CUE: The superposition principle, in Proceedings of the 2013 Physics Education Research Conference, Portland, OR, edited by P. V. Engelhardt, A. D. Churukian, and D. L. Jones (American Association of Physics Teachers, New York, 2014), pp. 385-388, https://doi.org/10.1119/perc.2013.pr.084.

[25] J. P. Zwolak and C. A. Manogue, Revealing differences between curricula using the colorado upper-division electrostatics diagnostic, in Proceedings of the 2014 Physics Education Research Conference, Minneapolis, $M N$, edited by P. V. Engelhardt, A. D. Churukian, and D. L. Jones (American Association of Physics Teachers, New York, 2015), pp. 295-298, https://doi.org/10.1119/ perc.2014.pr.070.

[26] E. Gire and E. Price, Arrows as anchors: An analysis of the material features of electric field vector arrows, Phys. Rev. ST Phys. Educ. Res. 10, 020112 (2014).

[27] S. V. Chasteen, S. J. Pollock, R. E. Pepper, and K. K. Perkins, Transforming the junior level: Outcomes from instruction and research in E\&M, Phys. Rev. ST Phys. Educ. Res. 8, 020107 (2012).

[28] P. Heller and M. Hollabaugh, Teaching problem solving through cooperative grouping. Part 2: Designing problems and structuring groups, Am. J. Phys. 60, 637 (1992), https://doi.org/10.1119/1.17118.

[29] E. van Zee and C. Manogue, A Study of the Development of the Paradigms in Physics Program, https://physics .oregonstate.edu/mathbook/P20, accessed: 2019/10/18.

[30] J. D. Bransford, A. L. Brown, and R. R. Cocking, How People Learn (National Academies Press, Washington, DC, 2000), ISBN 978-0-309-07036-2.

[31] J. L. Docktor and J. P. Mestre, Synthesis of disciplinebased education research in physics, Phys. Rev. ST Phys. Educ. Res. 10, 020119 (2014). 
[32] M. E. Loverude and B.S. Ambrose, Editorial: Focused collection: PER in upper-division physics courses, Phys. Rev. ST Phys. Educ. Res. 11, 020002 (2015).

[33] L. Vygotsky, Mind in Society: The Development of Higher Psychological Processes (Harvard University Press, Cambridge, England, 1980), ISBN 9780674076686.

[34] P. Cobb and E. Yackel, Constructivist, emergent, and sociocultural perspectives in the context of developmental research, Educ. Psychol. 31, 175 (1996).

[35] M. Cole, Cultural Psychology: A Once and Future Discipline (Harvard University Press, Cambridge, MA, 1996), ISBN 9780674179561.

[36] E. Wenger, Communities of Practice: Learning, Meaning, and Identity (Cambridge University Press, Cambridge, England, 1998), ISBN 9780521663632.

[37] R. R. Hake, Interactive-engagement versus traditional methods: A six-thousand-student survey of mechanics test data for introductory physics courses, Am. J. Phys. 66, 64 (1998).

[38] S. Freeman, S. L. Eddy, M. McDonough, M. K. Smith, N. Okoroafor, H. Jordt, and M. P. Wenderoth, Active learning increases student performance in science, engineering, and mathematics, Proc. Natl. Acad. Sci. U.S.A. 111, 8410 (2014).

[39] C. Kontra, S. Goldin-Meadow, and S. L. Beilock, Embodied learning across the life span, Topics Cogn. Sci. 4, 731 (2012).

[40] E. F. Redish, Teaching Physics with the Physics Suite (John Wiley \& Sons, New York, 2003), ISBN 9780471393788.

[41] E. Hutchins, Cognition in the Wild (MIT Press, Cambridge, MA, 1995).

[42] D. Tall and S. Vinner, Concept image and concept definition in mathematics with particular reference to limits and continuity, Educ. Studies Math. 12, 151 (1981).

[43] G. A. Miller, The magical number seven, plus or minus two: some limits on our capacity for processing information, Psychol. Rev. 63, 81 (1956).

[44] Cognitive Task Analysis, edited by J. M. Schraagen, S. F. Chipman, and V. Shalin (Psychology Press, Hove, East Sussex, UK, 2000).

[45] M. B. Kustusch, D. Roundy, T. Dray, and C. A. Manogue, Partial derivative games in thermodynamics: A cognitive task analysis, Phys. Rev. ST Phys. Educ. Res. 10, 010101 (2014).

[46] C. Turpen and N.D. Finkelstein, The construction of different classroom norms during Peer Instruction: Students perceive differences, Phys. Rev. ST Phys. Educ. Res. 6, 020123 (2010).

[47] D. Wood, J. S. Bruner, and G. Ross, The role of tutoring in problem solving, J. Child Psychol. Psychiatry 17, 89 (1976).

[48] J. Anghileri, Scaffolding practices that enhance mathematics learning, J. Math. Teach. Educ. 9, 33 (2006).

[49] I. Esmonde, Ideas and identities: Supporting equity in cooperative mathematics learning, Rev. Educ. Res. 79, 1008 (2009).

[50] C. Henderson and M. H. Dancy, Barriers to the use of research-based instructional strategies: The influence of both individual and situational characteristics, Phys. Rev. ST Phys. Educ. Res. 3, 020102 (2007).
[51] M. Dancy and C. Henderson, Framework for articulating instructional practices and conceptions, Phys. Rev. ST Phys. Educ. Res. 3, 010103 (2007).

[52] C. Henderson and M. Dancy, Impact of physics education research on the teaching of introductory quantitative physics in the United States, Phys. Rev. ST Phys. Educ. Res. 5, 020107 (2009).

[53] M. Dancy and C. Henderson, Pedagogical practices and instructional change of physics faculty, Am. J. Phys. 78, 1056 (2010).

[54] C. Henderson, M. Dancy, and M. Niewiadomska-Bugaj, Editorial: Physical review in physics education research 2.0, Phys. Rev. ST Phys. Educ. Res. 8, 1 (2012).

[55] C. Turpen, M. Dancy, and C. Henderson, Perceived affordances and constraints regarding instructors' use of Peer Instruction: Implications for promoting instructional change, Phys. Rev. Phys. Educ. Res. 12, 010116 (2016).

[56] C. Henderson and M. H. Dancy, Physics faculty and educational researchers: Divergent expectations as barriers to the diffusion of innovations, Am. J. Phys. 76, 79 (2008).

[57] C. Turpen and N.D. Finkelstein, Not all interactive engagement is the same: Variations in physics professors' implementation of Peer Instruction, Phys. Rev. ST Phys. Educ. Res. 5, 020101 (2009).

[58] Paradigms in Physics, Ring Cycle Sequence, https:// paradigms.oregonstate.edu/sequences/2/, accessed: 2020/ 01/30.

[59] D. J. Griffiths, Introduction to Electrodynamics, 4th ed. (Cambridge University Press, Cambridge, England, 2017), ISBN 978-1108420419.

[60] E. Gire, P. J. Emigh, K. H. Hahn, and M. Lenz, Making Sense of Physics Sensemaking, American Physical Society Forum on Education Newsletter (Fall 2018), https://www .aps.org/units/fed/newsletters/fall2018/making-sense.cfm.

[61] Paradigms in Physics, Curvilinear Coordinates Sequence, https://paradigms.oregonstate.edu/sequences/3/, accessed: 2020/01/30.

[62] The Vector Calculus Bridge Project, https://bridge.math .oregonstate.edu, accessed: 2019/10/18.

[63] Paradigms in Physics, Integration Sequence, https:// paradigms.oregonstate.edu/sequences/4/, accessed: 2020/ 01/30.

[64] T. Dray and C. A. Manogue, The Geometry of Static Fields, https://books.physics.oregonstate.edu/GSF/drvec.html, accessed: 2019/10/18.

[65] Paradigms in Physics, Power Series Sequence, https:// paradigms.oregonstate.edu/sequences/5/, accessed: 2020/ 01/30.

[66] Paradigms in Physics, Learning Progression on Partial Derivatives, https://paradigms.oregonstate.edu/progressions/ derivatives/, available: 2020/02/29.

[67] H. Chang, F. Ngunjiri, and K.-A. C. Hernandez, Collaborative Autoethnography (Taylor \& Francis Group, London, 2012).

[68] C. Henderson, A. Beach, and M. Famiano, Promoting instructional change via co-teaching, Am. J. Phys. 77, 274 (2009). 
[69] At OSU, the Paradigms are sometimes co-taught and have at least one graduate and/or undergraduate teaching assistant.

[70] M. Lenz, P. J. Emigh, and E. Gire, Surprise! students don't do special-case analysis when unaware of it, in Proceedings of the 2018 Physics Education Research Conference, Washington, D.C., edited by A. Traxler, Y. Cao, and S. Wolf (American Association of Physics Teachers, New York, 2019), https://doi.org/10.1119/ perc.2018.pr.Lenz.

[71] K. T. Hahn, P. J. Emigh, M. Lenz, and E. Gire, Student sense-making on homework in a sophomore mechanics course, in Proceedings of the 2017 Physics Education Research Conference Proceedings, Cincinnati, $\mathrm{OH}$, edited by L. Ding, A. Traxler, and Y. Cao (American Association of Physics Teachers, New York, 2018), pp. 160-163, https:// doi.org/10.1119/perc.2017.pr.035.
[72] A recent reorganization of the Paradigms, called Paradigms 2.0, has combined the two three-week Paradigms into a single five week Paradigm. We have chosen to discuss the original version in this article because we have more experience with it.

[73] L. McDermott, P. Shaffer (University of Washington Physics Education Group), Tutorials in Introductory Physics, No. v. 2, Prentice Hall Series in Educational Innovation (Prentice Hall, New York, 2002), ISBN 9780130662453.

[74] E. Mazur, Peer Instruction: A User's Manual, Pearson Series in Educational Innovation: Instructor Resources for Physics Series (Prentice Hall, New York, 1997), ISBN 9780135654415.

[75] D. R. Sokoloff, R. K. Thornton, and P. W. Laws, RealTime Physics: Active Learning Laboratories (Wiley, New York, 2011), ISBN 9780470768921. 